\title{
Fungal infection involvement in primary biliary cirrhosis: A review of 2 cases
}

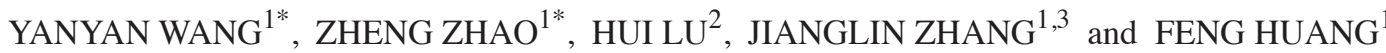 \\ ${ }^{1}$ Department of Rheumatology, Chinese PLA General Hospital, Beijing 100853; \\ ${ }^{2}$ Department of Nephrology, First Hospital Affiliated to General Hospital of PLA, Beijing 100048; \\ ${ }^{3}$ Department of Rheumatology, Chinese PLA General Hospital, Beijing 100853, P.R. China
}

Received October 3, 2014; Accepted November 7, 2016

DOI: $10.3892 /$ etm.2016.3977

\begin{abstract}
The present study aimed to analyze the imaging, clinical and pathological features of fungal infection involvement in primary biliary cirrhosis (PBC) by retrospectively analyzing and reviewing the features of two patients with fungal infection involvement in PBC. Both patients were female. One patient had a confirmed diagnosis of PBC. The other patient had confirmed Sjogren syndrome and PBC. The two cases of $\mathrm{PBC}$ were infected with fungal infection after treatment with hormonal and immunosuppressive agents. RCR of sputum confirmed Pneumocystis spp. infection in the patient with PBC alone. The mucormycosis infection was confirmed in the other patient after pathological examination of a renal biopsy. The state of the illnesses progressed quickly and both patients ultimately succumbed to their conditions. The patient prognosis of fungal infection involvement $\mathrm{PBC}$ is poor. Patients treated with long-term hormone and immunosuppressive agents should be monitored.
\end{abstract}

\section{Introduction}

Fungal infection has rarely been reported in patients with primary biliary cirrhosis (PBC); however it may occur following live transplant (LT) in patients with $\mathrm{PBC}$, predisposing factors for the development of fungal infection following LT include diabetes mellitus, cholestasis, hypertransfusion, acute rejection, treatment with high-dose of steroids and immunosuppressive agents, renal failure and bacterial infection (1). The present study aimed to analyze the imaging, clinical and pathological features of fungal infection involvement in $\mathrm{PBC}$

Correspondence to: Dr Jianglin Zhang, Department of Rheumatology, Chinese PLA General Hospital, 28 Fuxing Road, Beijing 100853, P.R. China

E-mail: jianglinzhangen@163.com

*Contributed equally

Key words: primary biliary cirrhosis, pneumocystis carinii pneumonia, mucormycosis by retrospectively analyzing and reviewing the features of two patients with fungal infection involvement in $\mathrm{PBC}$ who were admitted to our department.

\section{Case report}

Case 1. The present study was approved by the Ethics Committee of the Chinese PLA General Hospital and written informed consent was obtained from all patients. A 20 -year-old female presented with the following: Liver dysfunction lasting $>2$ years; xanthochromia lasting three months; and edema of the lower limb lasting one week, and was hospitalized in 2008. Liver dysdunction was confirmed in the patient following a physical examination in 2005; however, the patient refused standard treatment. In 2007, facial and yellow sclera and dark urine gradually developed. Following the administration of compound glycyrrhizin tablets (50 mg, 3/d; Minophagen Pharmaceutical Co., Ltd., Tokyo, Japan) and ademrtionine (500 mg, 3/d; Hospira UK Ltd., Hurley, UK), the patient suffered from a fever for five days; however, after terminating ingestion of the drugs, the fever was relieved. Abdominal distension, loss of appetite, dysfunction of liver, pancytopenia and ascites followed. A local hospital diagnosed the patient with sicca syndrome, cirrhosis and hypersplenism, and she was prescribed intravenous methylprednisolone (40 mg; 8 d; Pfizer Manufacturing Belgium, Puurs, Belgium), followed by implosive therapy of intravenous methylprednisolone $(1 \mathrm{~g} ; 1 \mathrm{~d})$. The dosage of intravenous methylprednisolone was maintained at $40 \mathrm{mg} / \mathrm{d}$, and the patient was treated with ursodeoxycholic acid (500 mg, 3/d; Losan Pharma GmbH, Neuenburg am Rhein, Germany) and glutathione (0.6 g; 3/d, Pharmainvest SPA, Boumedian, Algeria). The liver function of the patient was not greatly improved, and she presented with the following: petechia on both upper limbs; congestive rash on the palms; malaise; progressive decline of blood corpuscle; and edema below the lower limbs. Following progressive aggravation of her symptoms, the patient was admitted to our hospital.

Physical examination demonstrated the following: Moon face; symmetry congestive erythema on the face and hands; petechia and ecchymosis on the upper limbs (predominantly) and the nape of the neck; no jaundice of the skin; the superficial lymph node was not large and the lungs were normal; and hepatosplenomegaly, shifting dullness was negative. 
Laboratory tests results were conducted and the results were as follows (normal reference range): Routine blood tests: white blood cell count (WBC), 0.59 (3.5-10)x $10^{9} / 1$; red blood cell count (RBC), 3.12 (3.5-5.5) x10 $12 / 1$; hemoglobin $(\mathrm{Hb})$, 94 (110-176) g/l; and platelet count (PLT), 42×10\%/1. Routine urine tests: WBC, 2-3/HP; and urine protein (-). Routine stool tests: Helicobacter pylori, weakly positive; erythrocyte sedimentation rate (ESR), $11 \mathrm{~mm} / \mathrm{h}$; immunoglobin $\mathrm{G}$ (IgG), 1730 (700-1600) mg/dl; IgA 35.7 (70-400) mg/dl, C3, 43.2 (90-180) mg/dl; C4, 7.63 (10-40) mg/dl; and C-reactive protein (CRP), <0.308 (0-0.8) mg/dl. Autoantibody tests: Anti-nuclear antibody (ANA) (1:1,000 particles), positive, anti-SSA antibody, positive; anti-SSB antibody, positive; and anti-mitochondrial antibody (AMA), positive. Blood biochemistry tests: Albumin(Alb), 27.1 (35-50) g/l; total bilirubin(TBIL), 66.8 (0-21) $\mu \mathrm{mol} / \mathrm{l}$; direct bilirubin (DBIL), 48.9 (0-8.6)/mol/1; alanine aminotransferase (ALT), 296.7 (0-40) U/1; aspartate aminotransferase (AST), 109.5 (0-40) U/l; alkaline phosphatase (ALP), 244.9 (0-130) U/l; gamma glutamyl transferase (GGT), 213.1 (0-50) U/1; lactate dehydrogenase, 346.6 (0-200) U/1; glucose (GLU) $9.07 \mathrm{mmol} / \mathrm{l}$; blood urea nitrogen (BUN), 7.35 (1.8-7.5) mmol/1; creatinine (Cr), 76 (30-110) $\mu \mathrm{mol} / \mathrm{l}$; cancer antigen (CA) 125, 421.3 U/ml; CA19-9, $455.7 \mathrm{U} / \mathrm{ml}$; and blood ammonia, $92.1 \mu \mathrm{mol} / \mathrm{l}$. Purified protein derivative (PPD) test demonstrated and tuberculosis antibody were negative (1). Abdominal ultrasound demonstrated liver cirrhosis, splenomegaly and a small amount of ascites. Magnetic resonance (MR) imaging of the abdomen also indicated liver cirrhosis, splenomegaly, a small amount of ascites (Fig. 1).

Following admission to our hospital, the patient suffered from a cough producing white sputum. Lung CT tests showed that the upper lung was shadowed, and the possibility of tuberculosis was not excluded. Moxifloxacin hydrochloride (0.4 g; 1/d; Bayer AG, Leverkusen, Germany), streptomycin (0.75 g; im; 1/d; Merro Pharmaceutical Co., Ltd., Dalian, China) and ethambutol (0.75 g; 1/d; China Resources Double-Crane Pharmaceutical Co., Ltd., Beijing, China) were administered; however, the symptoms did not improve. The patient subsequently presented with wheezing, shortness of breath and difficult breathing. Blood gas analysis suggested hypoxemia and respiratory alkalosis. Lung CT scans indicated high-density imaging of the full lung (Fig. 2). Serum $\beta$-glucan test was positive and polymerase chain reaction analysis of the sputum detected inclusion bodies of Pneumocystis spp. The patient was treated with caspofungin acetate $(70 \mathrm{mg}$; i.v.; 1/d; MSD; Merck Millipore, Darmstadt, Germany); two slices of cotrimoxazole (3/d; Southwest Synthetic Pharmaceutical Co., Ltd., Chongqing, China) and bedside hemofiltration. The patient's condition became progressively aggravated and a chest radiograph showed pneumonia of both upper lungs (Fig. 3). Even with anti-infective treatment, the patient eventually succumbed to the severity of her condition. The final diagnosis was PBC and pneumonia caused by Pneumocystis carinii, which led to respiratory and circulatory failure.

Case 2. A 49-year-old female presented with intermittent fever and abdominal distension for more than a year. The patient was admitted to our hospital in 2006. The patient suffered from an intermittent fever without any known cause or regularity a year prior to admission (body temperature, $38^{\circ} \mathrm{C}$ ). Fever

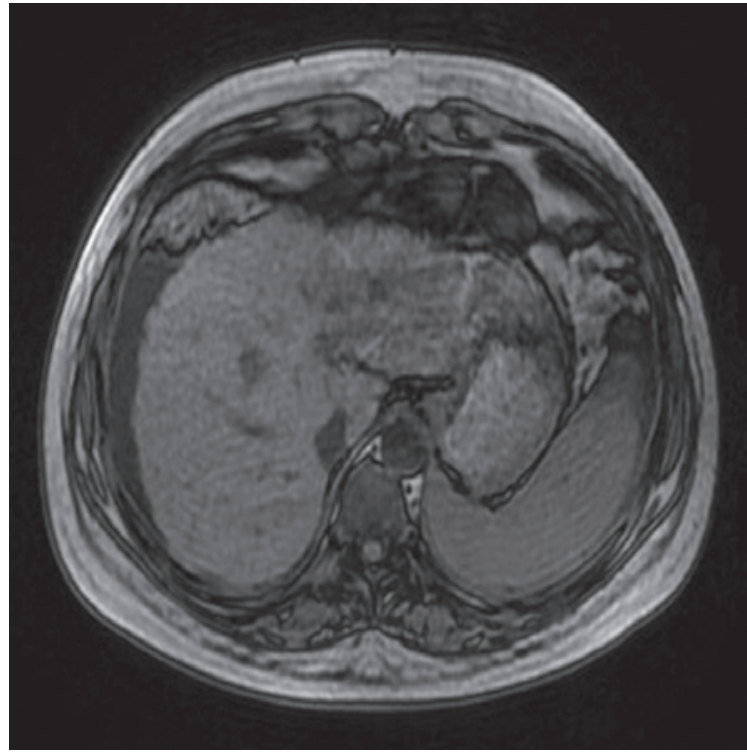

Figure 1. Case 1: Abdominal magnetic resonance scanning demonstrated cirrhosis with high density hypersplenotrophy splenomegaly.

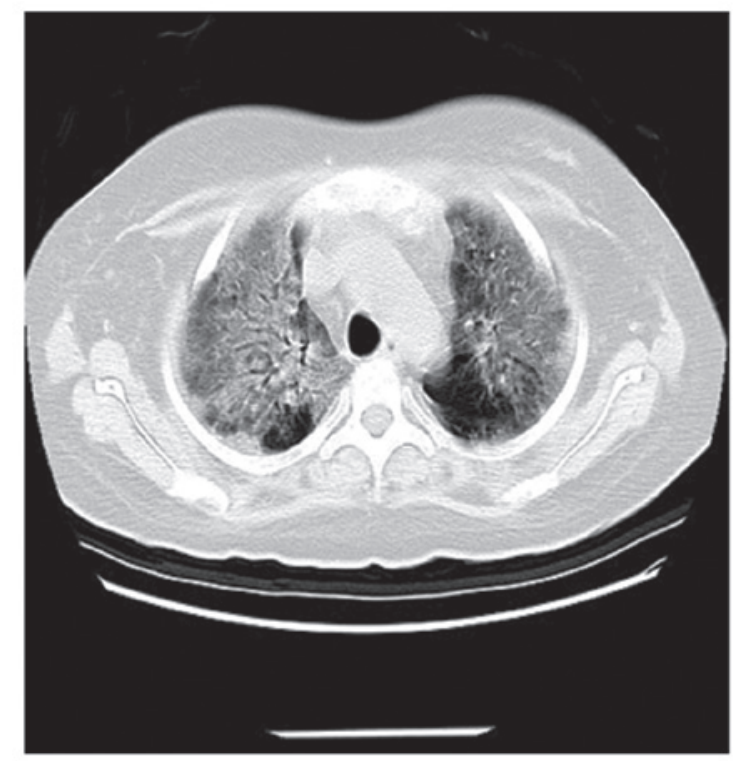

Figure 2. Case 1: Chest computed tomography scanning of the lungs.

was accompanied by fatigue, anorexia, cough, yellow sputum, night sweats and abdominal distension. ANA was positive, anti-dsDNA was confirmed as weakly positive and liver transaminase levels were mildly elevated. The patient was diagnosed with autoimmune hepatitis and was treated using compound glycyrrhizin tablets (50 mg; 3/d) and alprostadil (20 mg; i.v.; 1/d; Beijing TIDE Pharmaceutical Co., Ltd., Beijing, China), the patient was administered hepatic treatment. The patient's condition did not improve, rather it gradually worsened as her temperature reached up to $40^{\circ} \mathrm{C}$, peaking in the afternoon. Anti-smooth muscle antibody and AMA were positive. Taking the autoimmune cirrhosis and pulmonary infection into consideration, hepatic and anti-inflammatory symptomatic treatment consisting of ursodeoxycholic acid and cefuroxime axetil tablets $(0.5 \mathrm{~g}, 2 / \mathrm{d}$; GlaxoSmithKline, Brentford, UK) 


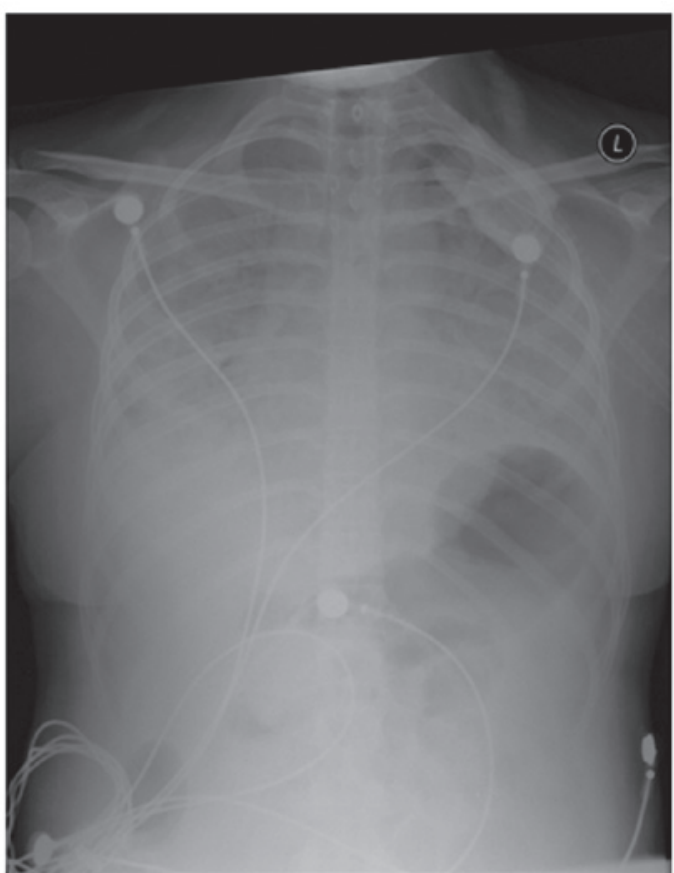

Figure 3. Case 1: Chest $\mathrm{X}$ ray demonstrated inflammation of both lungs, cirrhosis and hypersplenotrophy splenomegaly.

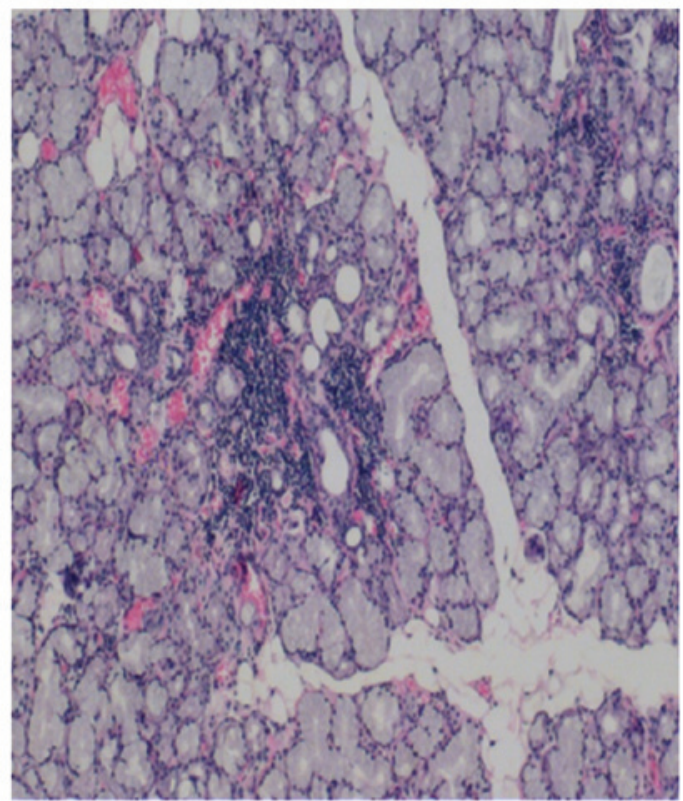

Figure 4. Case 2: Labial gland biopsy demonstrated focal lymphocytic infiltration.

was administered, and the symptoms of cough and expectoration eased but were accompanied by an intermittent fever. One week prior to hospital admission, hypogastrium pain (persistent dull pain) appeared with no nausea, vomiting, diarrhea or other symptoms. During the course of the disease, symptoms of thirst and joint pain were noted; however no obvious limitations in ingesting dry food were detected. The patient did not suffer from dry eyes, muscle pain, muscle weakness, facial butterfly spots or photophobia. The patient suffered from tuberculosis for 12 years, which was subsequently been cured.
Physical examination revealed scattered spider angioma on the anterior part of the neck and the left palm and mild pitting edema of the lower limbs. No other abnormalities were found. Laboratory test results were as follows: ANA autoantibodies (1:1,000 homogeneous particles), positive; anti-dsDNA, positive; anti-SSA antibody, negative; anti-SSB antibody, negative; IgA, 853 (70-400) mg/dl; IgG, 2820 (700-1600) mg/dl; IgM, 924 (40-230) mg/dl; IgE, 1400 (0-100) IU/ml; C3, 79.3 mg/dl; C4, $17.6 \mathrm{mg} / \mathrm{dl}$; and RF, 5410 (0-20) IU/ml. Routine blood tests:

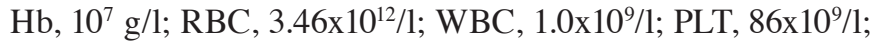
ESR, $129(<20) \mathrm{mm} / \mathrm{h}$; and CRP, <.313 mg/dl. Routine urine tests revealed a normal range and tests for hepatitis $\mathrm{B}$ and $\mathrm{C}$ and HIV were detected negative.

Blood biochemistry results were as follows: Alb, 32.9 (35-50) g/l; TBIL, 33.9 umol/1; DBIL, 19.4 umol/1; ALT, 35 U/l; AST, 84.1 U/1; ALP, 184.2 U/l; GGT, $140.8 \mathrm{U} / \mathrm{l}$; BUN, 2.42 (1.8-7.5) mmol/1; Cr, 38.8 (30-110) $\mu \mathrm{mol} / \mathrm{lU}$; $\mathrm{K}, 3.47$ (3.5-5.5) mmol/1, Ca, $2.20(2.25-2.75) \mathrm{mmol} / \mathrm{l}$; acid-fast stain (sputum), negative; tumor marker, negative. PPD test (1) demonstrated and tuberculosis antibody were negative. Sputum smear exhibited Gram-negative cocci and Gram-negative bacteria. Schirmer's test was $0 \mathrm{~cm}$; labial gland biopsy showed interstitial multifocal lymphocytic infiltration of the salivary gland tissue (Fig. 4). Bone marrow aspiration revealed hyperplasia of marrow activity, megakaryocytes were visible. Abdominal ultrasound indicated cirrhosis, splenauxe, portal hypertension, gallbladder wall thickening, echo nodules next to the spleen, an accessory spleen and small amount of ascites. The kidneys were normal.

Plain CT scanning of the lung revealed bilateral patchy high-density shadows, the boundary was unclear. In addition to changes in the lungs that were indicative of inflammation, several lymph glands were seen in the bilateral oxters and mediastinum.

The patient was diagnosed with Sjogren's syndrome, PBC and hypersplenism. She was treated with hormones (methylprednisolone; $36 \mathrm{mg} / \mathrm{d}$ ) immunosuppressants (mycophenolate; 1 g; 2/d; Roche Pharma Inc., Basel, Switzerland), ursodeoxycholic acid $(0.25 \mathrm{~g} ; 3 / \mathrm{d})$, and polyene phosphatidylcholine capsules (456 mg, 3/d; Sanofi-aventis Hong Kong Ltd., Hong Kong, China) and cefuroxime sodium for injection (1.5 g, $2 / d)$. Following treatment, the patient's body temperature dropped to within normal levels. Compared to the symptoms exhibited upon admission to our hospital, the patient's upper abdominal pain was markedly reduced. However, 14 days after she was admitted to hospital, routine urine tests indicated the following: WBC15, 18/HP; PRO, negative; $80 \%$ of erythrocytes were abnormally formed and $20 \%$ were uniform. Following this, gross hematuria appeared, the offside costalpinal angle exhibited tenderness and percussion pain, and pitting edema was confirmed in the patient's lower limbs. Renal ultrasound showed offside hydronephrosis accompanied by upside expansion of the offside ureter. Flocculent echo was detected in the right renal pelvis, which was indicative of a blood clot. Intravenous pyelography showed that the right kidney and ureter were undeveloped (Fig. 5). MR urograph demonstrated $5 \times 6 \mathrm{~cm}$ flakiness imaging in the parenchyma of right kidney. The kidney MRI and dynamic contrast-enhanced scan suggested cirrhosis, splenomegaly, a little ascites with abnormality of the right kidney (Fig. 6). 


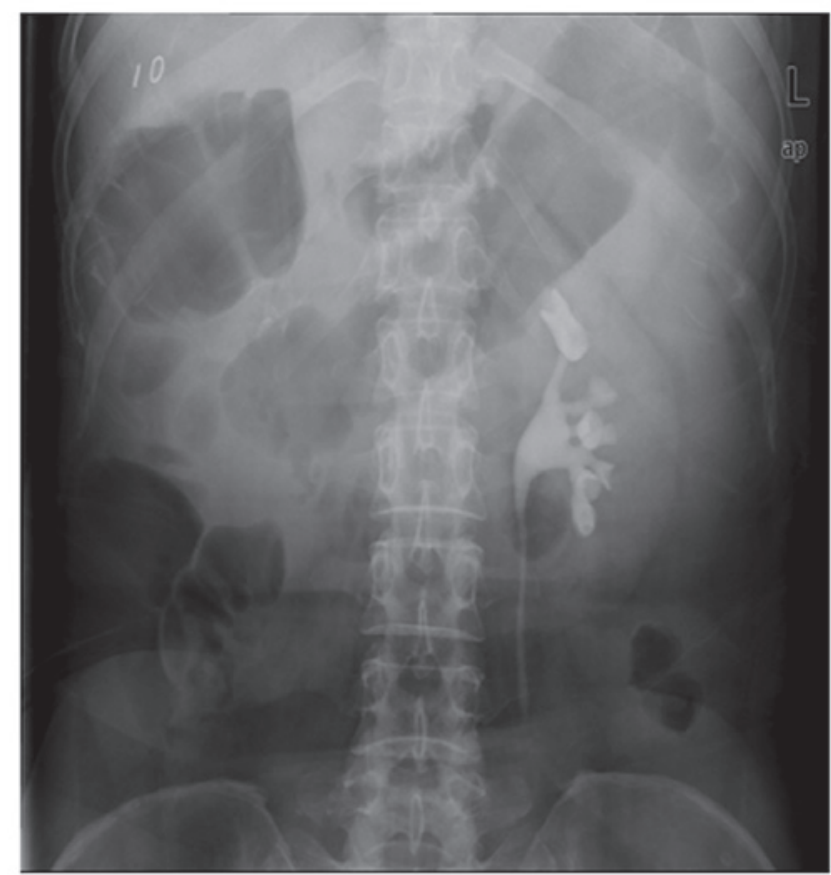

Figure 5. The right kidney and ureter were not visualized on intravenous urography until $60 \mathrm{~min}$. The left kidney and ureter revealed normal.

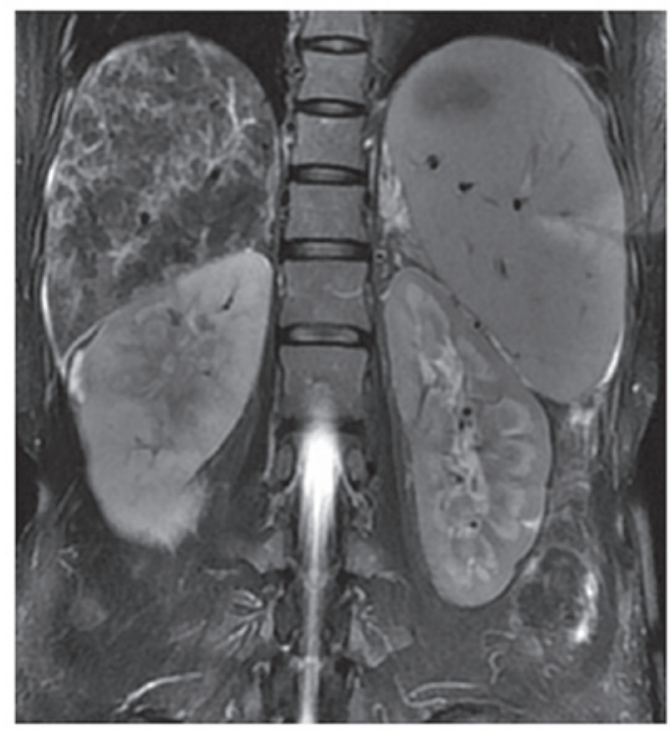

Figure 6. Case 2: T2-weighted magnetic resonance imaging of the kidney demonstrated cirrhosis and hypersplenotrophy splenomegaly abnormal changes in the right kidney.

The patient subsequently suffered from sudden impaired vision of the left eye, hyperemia of bulbar conjunctiva, photophobia and lacrimation. An eye examination indicated hypopyon of the left eye, the liquid was at the height of $\sim 1 / 5$ of the anterior chamber, cellulose before cystals of floating liquid material of aqueous humour (+++) exuded and vitreous opacities appeared. The patient was diagnosed with hypopyon of the left eye and uveitis of the left eye was confirmed. Atropine timolol (1\%; 1/d; Santen Pharmaceutical Co., Ltd., Osaka, Japan) was administered into the left eye and a dexamethasone and gentamicin mixture was inserted

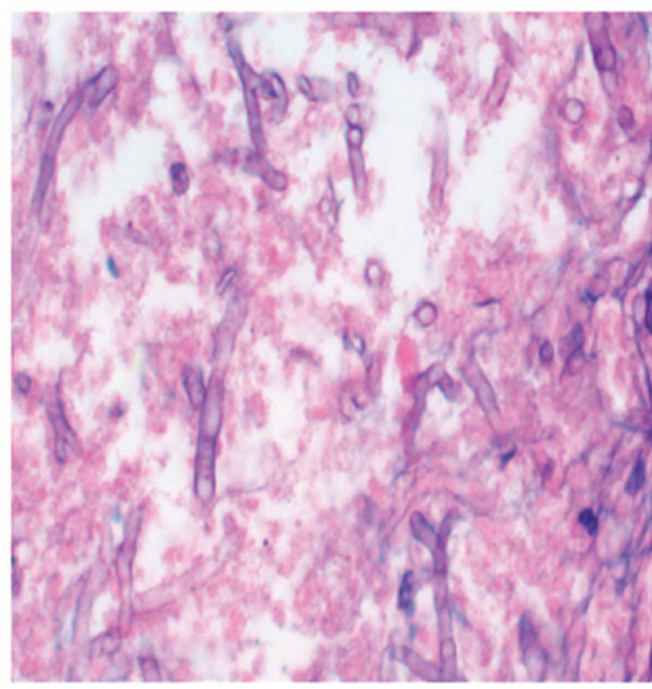

Figure 7. Case 2: Mucoraceous hyphae were detected (magnification, x400).

next to the left eye ball; however, the patient's vision did not markedly improved. To identify the nature of the disease, a renal biopsy was conducted under ultrasound guidance. The results suggested a renal mucormycosis infection (Fig. 7). The patient was provided with active anti-fungal amphotericin $\mathrm{B}$ therapy ( $2 \mathrm{mg}$, IV, 1/d; Northeast Pharmaceutical Group Co., Ltd., Shenyang, China) and nutritional support [human serum albumin (10 g, 1/d, Shanghai RAAS, Shanghai, China]. Due to the severity of the illness, the patient succumbed to the condition. The final diagnosis was Sjogren's syndrome leading to $\mathrm{PBC}$ and mucormycosis.

\section{Discussion}

PBC is a chronic intrahepatic cholestatic hepatopathy, the cause of which remains unclear. This condition predominantly occurs in middle-aged women and is characterized by progressive bile duct destruction in the liver, inflammation and fibrosis in the portal area and around the periportal, and eventual portal hypertension, liver cirrhosis and liver failure (2). With advances in diagnostic techniques and knowledge of the disease in recent years, the incidence rates of this disease have increased in China (3).

$\mathrm{PBC}$ is an autoimmune disease caused by immune system abnormalities. Previous studies have confirmed that PBC and other autoimmune diseases, such as Sjogren's syndrome and rheumatoid arthritis, have a common immunopathologic basis $(4,5)$. There is currently a lack of satisfactory therapies for the disease, as symptomatic treatment remains the main therapy option. Ursodeoxycholic acid has been successful in some patients for the relief of symptoms and has been shown to improve the indicators of liver function, delay disease progression and improve quality of life (6). If the patient has an additional connective tissue diseases, the patient is required to take hormones and immunosuppressive therapy simultaneously. Long-term use of hormones and immunosuppressive agents may increase the patient's risk of developing a variety of concurrent infections, particularly opportunistic infections $(7,8)$.

In the present study, two cases of fungal infection involvement in PBC, which was diagnosed in line with American 
Association for the Study of Liver Disease's PBC diagnosis recommendations published in 2000 (9). In case 1, the patient suffered from subsequent respiratory and cardiac failure; in case 2, the patient's condition was complicated by Sjogren's syndrome. Both of the patients were receiving hormonal and immunosuppressive therapy due to $\mathrm{PBC}$ prior to the development of concurrent $P$. carinii and mucormycosis infection. $P$. carinii infection and mucormycosis infection are opportunistic infections.

P. carinii pneumonia (PCP) is an opportunistic infectious disease. Individuals of any age can be infected with PCP, and people with normal immune function do not typically exhibit symptoms after infection. However, when the host's immune system is weakened, pneumocystis bacteria begin to multiply, and spread in the lungs, resulting in interstitial plasma cell pneumonia. Patients with connective tissue disease are at an increased risk of infection with $\mathrm{PCP}$ as they receive large doses of hormonal and immunosuppressive agents for long periods of time (10). In recent years, with the extensive use of biological agents, the incidence of fungal infections has significantly increased (11-14). In Japan, the rate of rheumatoid arthritis (RA) involvement in PCP infection after applying infliximab treatment is $0.4 \%$, at an average of 8.5 weeks after the application of infliximab (15). Rituximab monoclonal antibody treatment of RA and Wegener's granulomatosis (WG) has also been demonstrated to increase involvement in PCP (16).

Numerous clinical studies have reported systemic lupus erythematosus and WG involvement PCP infection (16-18). Reports of WG involvement in PCP infection are most common. In a meta-analysis of 11,905 cases of patients with connective tissue disease, $12 \%$ of the 578 cases of WG were complicated by PCP infection, whereas dermatomyositis/polymyositis involvement in PCP infection was only 6\%, SLE was $5 \%$ and RA was $1 \%$ (19). Connective tissue disease patients' infection with fungi and PCP is not only related to immune disorders of connective tissue disease but is also associated with the long-term use of hormonal and immunosuppressive therapy (20). However, it is difficult to elucidate which factor specifically causes the disease. In the present study, the two cases exhibited leukopenia upon admission to our hospital, as leukopenia may increase the chance of opportunistic infection.

Connective tissue disease involvement in PCP infection is related to treatment with cyclophosphamide, methotrexate, hormones, azathioprine, cyclosporine and other biological agents, such as anti-TNF inhibitors and anti-CD20 inhibitors $(21,22)$. Hormone therapy is the uppermost risk factor; $>90 \%$ of patients who present with fungal infection involvement PCP have received long-term hormone therapy (23). If elderly patients, patients with nutritional deficiency or patients with low-lymphocytes lipoproteinaemia receive $>16 \mathrm{mg} / \mathrm{d}$ equivalent hormone ( $>2$ months) or $>20 \mathrm{mg} / \mathrm{d}$ (>1-month) hormone therapy, the risk of PCP infection markedly increases (24). Cyclophosphamide treatment involvement in PCP infection may be related to significant inhibition of the lymphocyte count; therefore, patients regularly treated with cyclophosphamide should consider monitoring their CD4+ lymphocyte count.

To the best of our knowledge, there are no previous reports of $\mathrm{PBC}$ involvement in renal mucormycosis infection. In a clinical setting, there have been a small number of reports about liver involvement in mucormycosis infection after transplantation; the majority involve lung mucormycosis infection and reports of renal mucormycosis infection are rare (25). Mucormycosis describes various serious opportunistic infections caused by the following strains: Mucor spp., Absidia spp., Rhizopus spp. and Rhizomucor spp. which belong to the order of Mucoraceae (26). As the incidence rate is low, diagnosis remains difficult. Patients with the disease are predominantly complicated by diabetic acidosis, malnutrition, severe burns, trauma, leukemia, lymphoma, acquired immune deficiency syndrome or other serious wasting diseases, or have received long-term immunosuppressive agents, cytotoxic drugs or adrenocortical hormone (27). Most of the mucormycosis diseases are dangerous and the mortality rates are high (28).

The main feature of mucormycosis is sexual propagation producing zygosperm and asexual reproduction forming sporangia. Features include: i) hyphae are relatively thick, unseparated or rarely separated; ii) the mycelium wall is relatively thick, the side shoot is at right angle with the base shoot; and iii) tend to violate the vessel wall and lumen, thus forming a vessel clot that leads to ischemia, hemorrhage infarction and necrotizing inflammation of adjacent tissues (29). The clinical manifestations of mucor infections are cerebral, lung, gastrointestinal, cutaneous and systemically-spreading infections. The symptoms of patients with acute disease progress quickly and patients often succumb to the disease within several days or several weeks (30). Chronic infection can be manifested as simple granuloma or pyogenic inflammation and granulomatous mixed inflammation $(31,32)$. Clinical diagnosis is often based on clinical symptoms, susceptible factors, mycological examination and pathological biopsy, of which, locating hypha in pathological tissue is of most diagnostic significance. There are various reports of pulmonary mucormycosis imaging (33). Its initial performance is unilateral or bilateral bronchial pneumonia integrating into a large consolidation rapidly and often forming porosis. Single or multiple nodules can also be detected. If the pulmonary embolism is relatively large, the wedge-shaped shadow close to the pleura at the bottom can be seen, which has diagnostic significance. High-resolution CT can detect symptoms that cannot be seen on an X-ray, such as lumen blocking, vignette and pulmonary artery pseudoaneurysm caused by mucormycosis within the bronchus. It has not yet been described in the imaging literature of mucor infection. Mucormycosis progresses rapidly, therefore, early diagnosis and effective treatment are of decisive significance for the prognosis of patients with mucormycosis.

Renal involvement of systemically disseminated mucormycosis is rare, and simple renal mucormycosis is scarcer. Case 2 received hormonal and immunosuppressive therapy due to Sjogren's syndrome involvement in PBC. Following therapy, the patient was infected with renal mucormycosis. Despite active amphotericin B anti-infective therapy, the course of disease progressed quickly, and lead to mortality in this patient.

The present study provided a retrospective analysis of two cases of PBC involvement in opportunistic fungal infections. The patients in these cases were infected with fungi after taking hormones and immunosuppressive agents for a long 
period of time. This suggests that in patients with rheumatic autoimmune diseases, who receive long-term hormonal and immunosuppressive therapy, the probability of opportunistic pathogen infections increases. These findings are of particular use in clinical situations. When confronted with an infection whose response to antibiotic therapy is not ideal, a sputum smear and tests for fungi should be conducted. When necessary, an early histopathological biopsy should be performed to make time for the treatment of the patient. At the same time, actively seeking pathological diagnosis will improve our understanding of these difficult diseases.

\section{References}

1. Davari HR, Malekhossini SA, Salahi HA, Bahador A, Saberifirozi M, Geramizadeh B, Lahsaee SM, Khosravi MB, Imanieh $\mathrm{MH}$ and Bagheri $\mathrm{MH}$ : Outcome of mucormycosis in liver transplantation: Four cases and a review of literature. Exp Clin Transplant 1: 147-152, 2003.

2. Liberal R and Grant CR: Cirrhosis and autoimmune liver disease: Current understanding. World J Hepatol 8: 1157-1168, 2016.

3. Liu H, Liu Y, Wang L, Xu D, Lin B, Zhong R, Gong S, Podda M and Invernizzi P: Prevalence of primary biliary cirrhosis in adults referring hospital for annual health check-up in Southern China. BMC Gastroenterol 10: 100, 2010.

4. Smyk DS, Bogdanos DP, Mytilinaiou MG, Burroughs AK and Rigopoulou EI: Rheumatoid arthritis and primary biliary cirrhosis: Cause, consequence, or coincidence? Arthritis 2012: 391567, 2012.

5. Selmi C, Meroni PL and Gershwin ME: Primary biliary cirrhosis and Sjögren's syndrome: Autoimmune epithelitis. J Autoimmun 39: 34-42, 2012

6. Poupon R: Ursodeoxycholic acid and bile-acid mimetics as therapeutic agents for cholestatic liver diseases: An overview of their mechanisms of action. Clin Res Hepatol Gastroenterol 36 (Suppl 1): S3-S12, 2012.

7. Mino Y: Optimization of immunosuppression and the prevention of fungal infection in autoimmune diseases. Yakugaku Zasshi 135: 1123-1127, 2015. (In Japanese)

8. Takizawa Y, Inokuma S, Tanaka Y, Saito K, Atsumi T, Hirakata M, Kameda H, Hirohata S, Kondo H, Kumagai $\mathrm{S}$ et al: Clinical characteristics of cytomegalovirus infection in rheumatic diseases: Multicentre survey in a large patient population. Rheumatology 47: 1373-1378, 2008.

9. Toda G: Revised criteria for diagnosis of autoimmune hepatitis Intern Med 39: 999-1000, 2000

10. Chen M, Tian X, Qin F, Zhou J, Liu J, Wang M and Xu KF: Pneumocystis pneumonia in patients with autoimmune diseases: A retrospective study focused on clinical characteristics and prognostic factors related to death. PLoS One 10 e0139144, 2015.

11. Wissmann G, Morilla R,Martín-GarridoI,Friaza V, Respaldiza N, Povedano J, Praena-Fernández JM, Montes-Cano MA, Medrano FJ, Goldani LZ, et al: Pneumocystis jirovecii colonization in patients treated with infliximab. Eur J Clin Invest 41: 343-348, 2011.

12. Takeuchi T and Kameda H: The Japanese experience with biologic therapies for rheumatoid arthritis. Nat Rev Rheumatol 6 : 644-652, 2010

13. Karino T, Osaki K, Kanamori K and Yahata T: Case of pulmonary cryptococcosis which developed in a patient receiving abatacept therapy for rheumatoid arthritis. Nihon Kokyuki Gakkai Zasshi 48: 980-984, 2010. (In Japanese).

14. Yamazaki H, Nanki T, Miyasaka N and Harigai M: Methotrexate and trimethoprim-sulfamethoxazole for Pneumocystis pneumonia prophylaxis. J Rheumatol 38: 777, author reply 778, 2011.
15. Kameda H, Tokuda H, Sakai F, Johkoh T, Mori S, Yoshida Y, Takayanagi N, Taki H, Hasegawa Y, Hatta K, et al: Clinical and radiological features of acute-onset diffuse interstitial lung diseases in patients with rheumatoid arthritis receiving treatment with biological agents: Importance of Pneumocystis pneumonia in Japan revealed by a multicenter study. Intern Med 50: 305-313, 2011.

16. Hugle B, Solomon M, Harvey E, James A, Wadhwa A, Amin R, Bell-Peter A and Benseler S: Pneumocystis jiroveci pneumonia following rituximab treatment in Wegener's granulomatosis. Arthritis Care Res 62: 1661-1664, 2010.

17. Weng CT, Liu MF, Weng MY, Lee NY, Wang MC, Lin WC, Ou CY, Lai WW, Hsu SC, Chao SC, et al: Pneumocystis jirovecii pneumonia in systemic lupus erythematosus from southern Taiwan. J Clin Rheumatol 19: 252-258, 2013.

18. Khellaf M and Godeau B: Pneumocystis pneumonia among patients with systemic diseases. Presse Med 38: 251-259, 2009. (In French)

19. Falagas ME, Manta KG, Betsi GI and Pappas G: Infection-related morbidity and mortality in patients with connective tissue diseases: A systematic review. Clin Rheumatol 26: 663-670, 2007.

20. Dilger K, Hohenester S, Winkler-Budenhofer U, Bastiaansen BA, Schaap FG, Rust C and Beuers U: Effect of ursodeoxycholic acid on bile acid profiles and intestinal detoxification machinery in primary biliary cirrhosis and health. J Hepatol 57: 133-140, 2012.

21. Ogawa J, Harigai M, Nagasaka K, Nakamura T and Miyasaka N: Prediction of and prophylaxis against Pneumocystis pneumonia in patients with connective tissue diseases undergoing mediumor high-dose corticosteroid therapy. Mod Rheumatol 15: 91-96, 2005 .

22. Jenks KA, Stamp LK, O'Donnell JL, Savage RL and Chapman PT: Leflunomide-associated infections in rheumatoid arthritis. J Rheumatol 34: 2201-2203, 2007.

23. Meuli K, Chapman P, O'Donnell J, Frampton C and Stamp L: Audit of pneumocystis pneumonia in patients seen by the Christchurch Hospital rheumatology service over a 5-year period. Intern Med J 37: 687-692, 2007.

24. Einollahi B, Lessan-Pezeshki M, Aslani J, Nemati E, Rostami Z, Hosseini MJ, Ghadiani MH, Ahmadpour P, Shahbazian H, Pour-Reza-Gholi F, et al: Two decades of experience in mucormycosis after kidney transplantation. Ann Transplant 16: 44-48, 2011.

25. Danion F, Aguilar C, Catherinot E, Alanio A, DeWolf S, Lortholary O and Lanternier F: Mucormycosis: New Developments into a Persistently Devastating Infection. Semin Respir Crit Care Med 36: 692-705, 2015.

26. Ribes JA, Vanover-Sams CL and Baker DJ: Zygomycetes in human disease. Clin Microbiol Rev 13: 236-301, 2000.

27. Langford S, Trubiano JA, Saxon S, Spelman D and Morrissey CO: Mucormycete infection or colonisation: Experience of an Australian tertiary referral centre. Mycoses 59: 291-295, 2016.

28. Guarner J and Brandt ME: Histopathologic diagnosis of fungal infections in the 21st century. Clin Microbiol Rev 24: 247-280, 2011.

29. Wu X, Xu G, Wen $\mathrm{W}$ and Guo J: Clinical study on aggressive rhinocerebral mucormycosis. Lin Chung Er Bi Yan Hou Tou Jing Wai Ke Za Zhi 22: 1060-1062, 2008. (In Chinese).

30. Vaezi A, Moazeni M, Rahimi MT, de Hoog S and Badali H: Mucormycosis in Iran: A systematic review. Mycoses 59: 402-415, 2016

31. Jung J, Kim MY, Lee HJ, Park YS, Lee SO, Choi SH, Kim YS, Woo JH and Kim SH: Comparison of computed tomographic findings in pulmonary mucormycosis and invasive pulmonary aspergillosis. Clin Microbiol Infect 21: e11-e18, 2015.

32. Nampoory MR, Khan ZU, Johny KV, Constandi JN, Gupta RK, Al-Muzairi I, Samhan M, Mozavi M and Chugh TD: Invasive fungal infections in renal transplant recipients. J Infect 33: 95-101, 1996.

33. Chkhotua A, Yussim A, Tovar A, Weinberger M, Sobolev V, Bar-Nathan N, Shaharabani E, Shapira Z and Mor E: Mucormycosis of the renal allograft: Case report and review of the literature. Transpl Int 14: 438-441, 2001. 